\title{
A novel method to estimate platelet counts from peripheral smears: A study comparing a new method of platelet estimation with existing methods
}

\author{
Muthu Sudalaimuthu*, Shivasekar Ganapathy, Koshalya Rajendran and Sundaram Arunachalam \\ SRM Medical College Hospital and Research Centre, Potheri, Kattankulathur, Kancheepuram District, TamilNadu, India
}

\section{ABSTRACT}

Background: Automated hematology analyzers have a low degree of accuracy in predicting platelet counts in patients with thrombocytopenia. Hence automated platelet counts should be crosschecked by reviewing the peripheral blood smear in cases of thrombocytopenia. However existing methods for platelet count estimation from peripheral blood smears have certain drawbacks. Hence we suggest a new method to estimate platelet count from peripheral smear and compared it with the existing methods.

Methods: Platelet count was estimated by four different methods in 200 blood samples and compared with the platelet count obtained from automated analyzer Sysmex XT-1800i. Method A was based on the platelet/Red Blood cell (RBC) ratio in ten oil immersion fields and multiplying it by total RBC count, Methods B, C and D were based on the average number of platelets per oil immersion field. Platelet count was obtained by multiplying by 15000 in Method B, by 20000 in Method C, by hemoglobin value X 1000 in Method D. Results were analyzed by ANOVA, student's t test and correlation coefficient.

Result: Results of Method A had a strong correlation with the automated blood counts (0.973). The results were not significantly different from the automated blood counts ( $\mathrm{p}$ value of 0.798 ). Results of other methods were significantly different from automated platelet counts $(\mathrm{p}<0.05)$.

Conclusion: Platelet count estimation based on platelet/RBC ratio in ten oil immersion fields and total $\mathrm{RBC}$ count is a simple and reliable method to estimate platelet counts from peripheral smears.

Keywords: Platelet, Platelet count, Thrombocytopenia, Hematology

\section{Introduction}

Automated hematology analyzers are routinely being used for performing complete blood counts. But the accuracy of this method in predicting the platelet count in patients with thrombocytopenia is questionable ${ }^{[1,2]}$. Hence there is a need for an alternate method to perform the platelet counts with reasonable degree of accuracy in patients with thrombocytopenia. The International Council for Standardization in Hematology (ICSH) and the International Society of Laboratory Hematology (ISLH) have recommended a method based on the measurement of platelet/RBC ratio with fluorescent labeled platelets in fluorescent flow cytometer as the reference method for platelet counting in peripheral blood ${ }^{[3]}$. But this method is expensive and cannot be performed routinely in developing countries. A traditional method for counting platelets in a peripheral smear which has been in use for a long time is by taking the average of platelets in ten oil immersion fields and multiplying it by 15000 or $20000^{[4,5]}$. But this method has its own drawbacks. Hence this study was performed to compare the various methods which can be used to manually estimate the platelet count in peripheral blood smear and to find out the accurate method among them.

\section{Materials And Methods}

Samples from 200 patients who visited our laboratory for performing complete blood counts were included in our study. An informed consent for doing this study was obtained from the patients. Venous blood from these patients was collected in tubes containing Ethylene Diamine Tetraacetic Acid. All these samples were analyzed within three hours after ruling out blood clots and hemolysis in the sample. Hemoglobin, Red Blood Cell (RBC) counts and platelet counts were obtained from the automated hematology analyzer Sysmex XT-1800i. Air dried thin peripheral blood smears were made from the samples and stained with Leishman stain. Peripheral blood smear was examined under the light microscope (Leica DM750). Smears with platelet clumps were excluded from the study.

Total number of platelets was counted in ten oil immersion fields at the junction of body and tail where the cells were in monolayer (neither overlapping nor widely spaced). Number of RBCs in these ten fields was also counted by 
counting the RBCs in one quarter of all oil immersion fields and multiplying it by four. Platelet count of the blood sample was calculated manually by four different methods as described in Table 1. Platelet counts obtained by the four methods were compared with the platelet count from automated hematology analyzer. ANOVA, student's t test, Pearson Product Moment correlation coefficient and linear regression analysis scatterplots were used to analyze the results from the different methods of platelet estimation. $\mathrm{p}$ value of $<0.05$ was considered significant difference in student's t test.

\section{Results}

Hemoglobin values of these samples ranged from 2.8 to $19.7 \mathrm{~g} / \mathrm{dL}$ while the RBC counts ranged from 0.8 to 6.4 million $/ \mu \mathrm{L}$. Platelet count from the automated analyzer ranged from 35000 to 607000 per $\mu \mathrm{L}$ with a mean of
249225.Platelet range and the mean value of the platelet counts from all the four methods is given in Table 2 .. ANOVA showed that the difference in the means of these methods was statistically significant. Student t test showed that the mean platelet count from Method A did not differ significantly from that of the automated analyzer ( $p$ value of 0.798). Method A and automated analyzer results had a high Pearson Product Moment correlation coefficient of 0.973 . Scatterplot depicting the results of these two methods showed only minimal dispersion (Fig 1). All these indicate that there is a strong agreement between platelet counting by Method A and the automated analyzer. Mean value of all the other methods differed significantly from that of the automated analyzer with $\mathrm{p}$ values ranging from 0.018 to $<0.001$ (Table 2). Their scatterplots also showed more dispersion than Method A when their results were plotted against that of automated analyzer (Figs 2-4).

Table 1: Methods of platelet estimation used in our study.

\begin{tabular}{|l|l|}
\hline Method & Formula used to calculate platelet count of the sample per $\mu \mathrm{L}$ \\
\hline Method A & $\frac{\text { Number of platelets in } 10 \text { OIF }}{\text { Number of RBCs in 10 OIF }}$ RBC Count/ $\mu \mathrm{L}$ \\
\hline Method B & $\frac{\text { Number of platelets in } 10 \text { OIF }}{10}$ X 15000 \\
\hline Method C & $\frac{\text { Number of platelets in } 10 \text { OIF }}{10}$ X 20000 \\
\hline Method D & $\frac{\text { Number of platelets in } 10 \text { OIF }}{10}$ X Hb in grams per dL X 1000 \\
\hline
\end{tabular}

OIF - Oil immersion field. RBC-Red blood cell. Hb-Hemoglobin

Table 2: Statistical data of the results

\begin{tabular}{|l|l|l|l|l|}
\hline & Method A & Method B & Method C & Method D \\
\hline Platelet count range $(p e r \mu \mathrm{L})$ & 39200 to 624000 & 42000 to 720000 & 56000 to 960000 & 8680 to 576000 \\
\hline Mean of the platelet counts & 251532 & 292522.5 & 390030 & 226677.4 \\
\hline $\begin{array}{l}\text { Difference from automated } \\
\text { analyzer (based on student's t } \\
\text { test } p \text { value) }\end{array}$ & $\begin{array}{l}\text { No statistically } \\
\text { significant difference } \\
(p \text { value 0.798) }\end{array}$ & $\begin{array}{l}\text { Statistically significant } \\
\text { difference } \\
(p \text { value }<0.001)\end{array}$ & $\begin{array}{l}\text { Statistically significant } \\
\text { difference } \\
\text { ( } p \text { value }<0.001)\end{array}$ & $\begin{array}{l}\text { Statistically significant } \\
\text { difference } \\
(p \text { value 0.018) }\end{array}$ \\
\hline Correlation coefficient & 0.973 & 0.944 & 0.944 & 0.789 \\
\hline
\end{tabular}

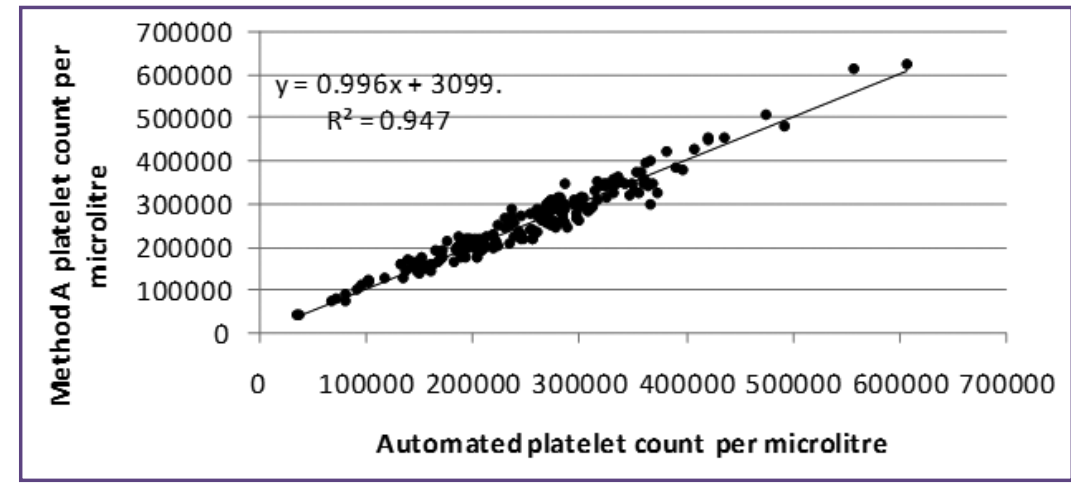

Fig. 1: Regression analysis scatterplot comparing Method A and automatic platelet counts showing only minimal dispersion. 


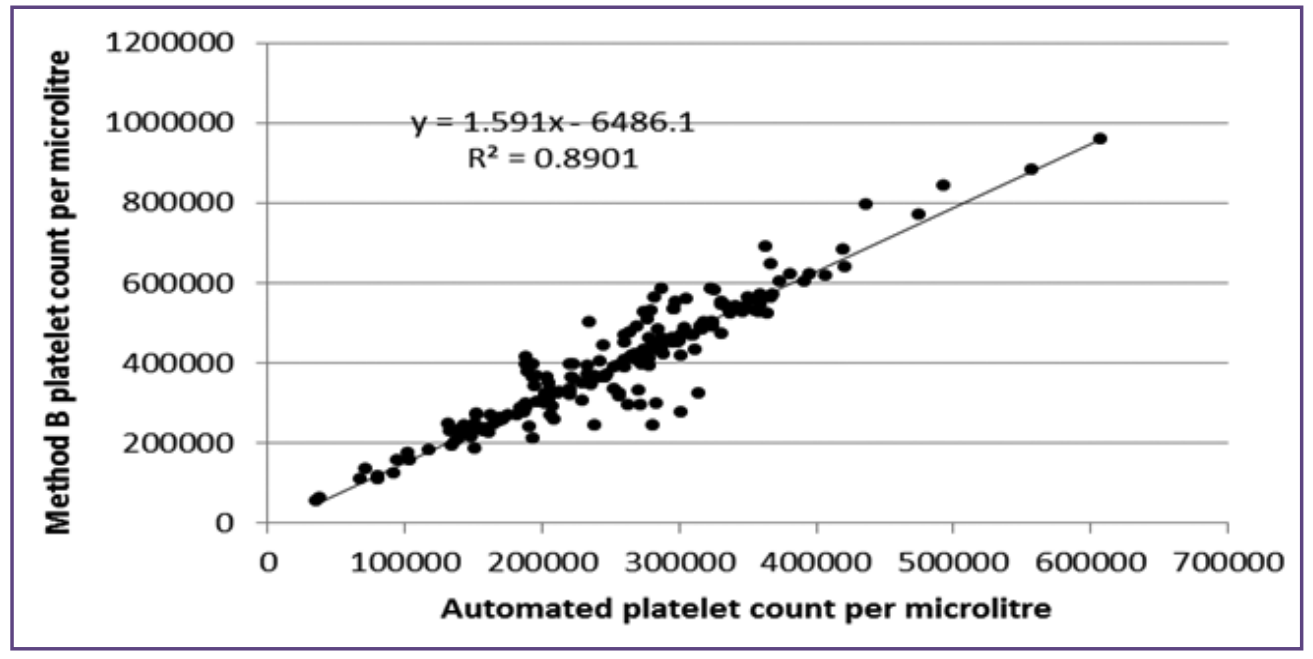

Fig. 2: Regression analysis scatterplot comparing Method B and automatic platelet counts showing moderate dispersion.

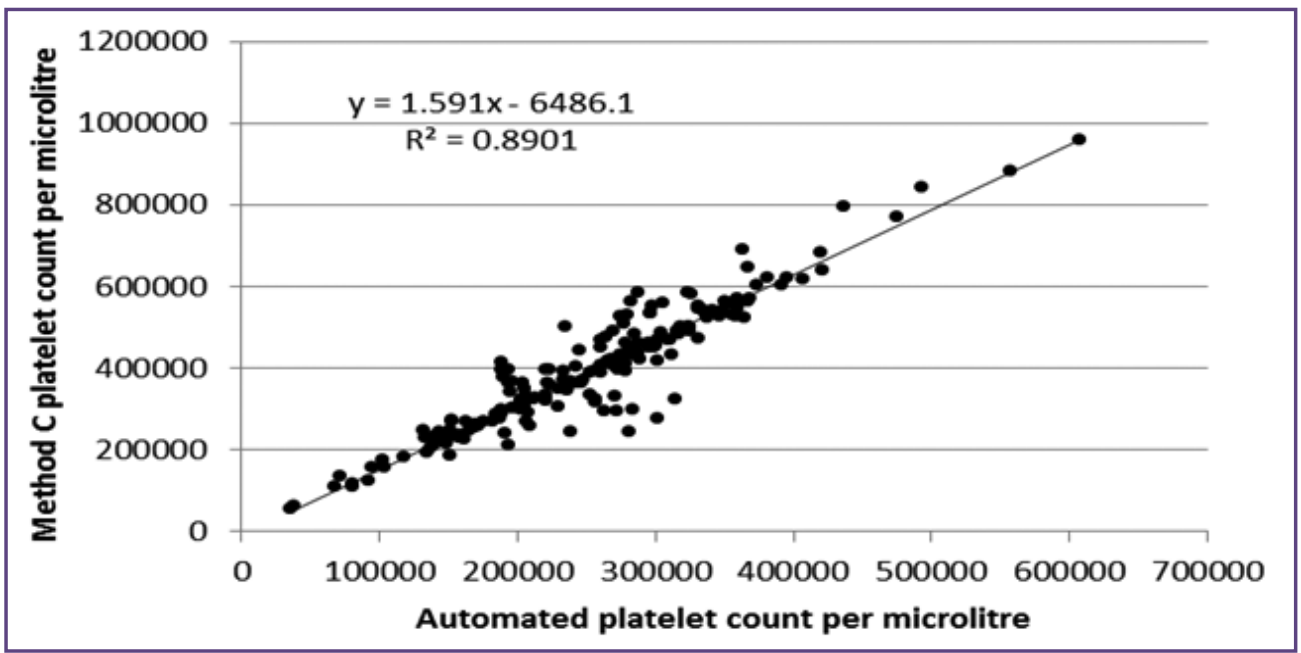

Fig. 3: Regression analysis scatterplot comparing Method C and automatic platelet counts showing moderate dispersion.

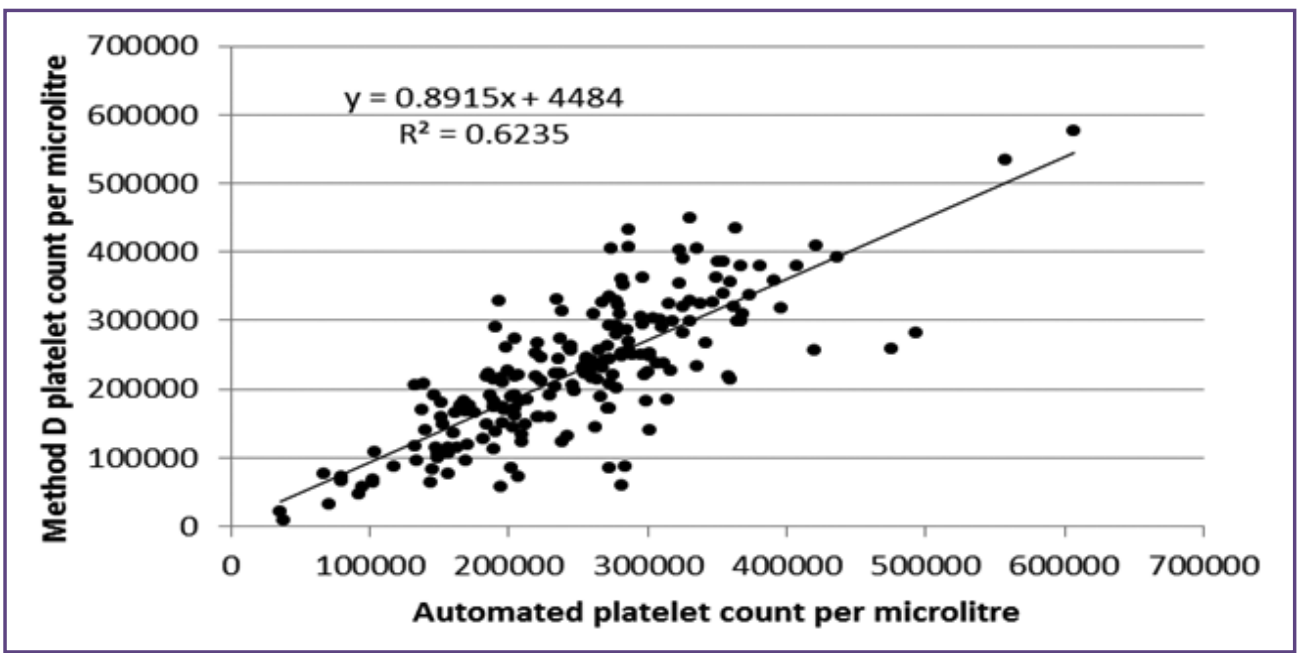

Fig. 4: Regression analysis scatterplot comparing Method D and automatic platelet counts showing wider dispersion. 


\section{Discussion}

Accurate measurement of the platelet counts is essential particularly in the management and monitoring of patients with thrombocytopenia ${ }^{[1,2,6]}$. Automated hematology analyzers in spite of the various advances have the limitation of inability to produce precise and reproducible platelet counts particularly in patients with thrombocytopenia $[1,2,6,7]$. Presence of background debris, microorganisms, fragmented RBCs, apoptotic bodies from white blood cells and markedly microcytic RBCs can all hinder an accurate measurement of platelets in automated hematology analyzers by mimicking the platelets due to their small size. On the other hand, platelet clumps and giant platelets owing to their large size are not counted as platelets in automated analyzers. All these lead to inaccurate platelet counts in an automated hematology analyzer ${ }^{[8-11]}$. Hence platelet count values obtained from automated analyzers should be crosschecked by some other method particularly in cases of thrombocytopenia ${ }^{[12]}$.

The traditional gold standard method for measuring platelet counts was the phase contrast microscopy ${ }^{[1]}$. But this method is time-consuming and lacks precision in cases with low platelet counts. The International Council for Standardization in Hematology (ICSH) and the International Society of Laboratory Hematology (ISLH) recommended a reference method for platelet counting in the year $2001^{[3]}$. This method measures the platelet count based on platelet/ $\mathrm{RBC}$ ratio and total $\mathrm{RBC}$ count. This method has been found to be very accurate ${ }^{[13]}$. But the procedure involves the detection of fluorescent labeled platelets in a fluorescence flow-cytometer which cannot be performed routinely in developing countries due to the costs involved. Hence the need for a simpler method to crosscheck the platelet counts from automated analyzers continues.

Brahimi et al ${ }^{[8]}$ suggested a method in 2008 in which they measured platelet count in peripheral smear by counting the number of platelets per $1000 \mathrm{RBCs}$ and multiplying it by total RBC count. Abid ${ }^{[14]}$ also showed similar findings in their study. This method resembled the International reference method recommended by ICSH and ISLH but is done manually in a peripheral smear. Their study results show that results from this method correlated well with the platelet count from automated analyzer. But when we applied the same method in our setup, we noticed that when the number of platelets were counted only for $1000 \mathrm{RBCs}$, many a times only three or four oil immersion fields could be counted as $1000 \mathrm{RBCs}$ were present in three or four fields itself. Counting the platelets in only three or four oil immersion fields in an entire peripheral smear is not ideal because they may not be representative fields of the entire smear. Hence we suggest an alternative method in which the number of platelets and RBCs have to be counted in ten oil immersion fields to calculate the platelet/RBC ratio. The value has to be multiplied by total $\mathrm{RBC}$ count to get the platelet count of the sample. To check the accuracy of this method, we took this method as Method A of platelet estimation in our study.

The method that is routinely used in many laboratories to verify the automated platelet count is by multiplying the average number of platelets per oil immersion field by 15000 or $20000^{[4,5]}$. Average number of platelets per field is calculated after counting ten oil immersion fields. A multiplication factor of 20000 is based on the study by Nosanchuk et al $^{[4]}$ in which he statistically validated the estimation of platelet count by counting platelets in peripheral smears. But later, Webb et al ${ }^{[5]}$ showed in 2004 that a multiplication factor of 15000 showed better results than a multiplication factor of 20000 in the same method. Uncertainty still exists as some pathologists use 20000 as multiplication factor while some others use $15000^{[15,16]}$. We selected the method with 15000 as multiplication factor as Method B and the method with 20000 as multiplication factor as Method C in our study.

Torres et al ${ }^{[17]}$ described a method in 2004 in which they multiplied the average number of platelets seen per field with the hemoglobin level of the patient and multiplied it by 1000 . They suggested from their study that this method is more specific than the traditional method of multiplying by 15000 or 20000 . This method was taken as Method D of platelet estimation in our study.

Our study results show that estimation of platelet counts based on platelet/ RBC ratio in ten fields (Method A) yields results comparable to that of the automated hematology analyzer. A strong correlation coefficient (0.973), minimal dispersion in the scatterplot when plotted against the automated analyzer values and a high $\mathrm{p}$ value in student's test - all these suggest that this method is an ideal but simple method to crosscheck the platelet count results of automated analyzer. But the disadvantage with this method it can be difficult in the beginning stage to count the RBCs. Hence we did the RBC count only in one quarter of all the ten oil immersion fields. These values from all the ten fields can be added and then multiplied by four to achieve the total number of RBCs in ten fields. RBC counting can be made easier by focusing something easily identifiable 
right in the center point of the field. This will help us in identifying the boundaries of the quadrant that we are counting. However in the case of platelets we counted them in all the four quadrants of each field. All these counting should be done at the junction of body and tail of the smear where the cells are in monolayer.

Results of Methods B (Average platelets per oil immersion field multiplied by 15000) and C (Average platelets per oil immersion field multiplied by 20000) differed significantly from that of the automated hematology analyzer $(\mathrm{p}<$ 0.001 ). When plotted against the automated platelet count, the scatterplots showed more degree of dispersion when compared to Method A. This is significant because this method is practiced in many laboratories to verify the automated platelet count. Our study results are in contrast to the results of some of the earlier studies which have shown these methods to correlate with automated platelet count ${ }^{[4,5]}$. As many people practicing this method would have noted, this method is not highly accurate and has inter-observer variability as well. This inter-observer variability can be minimized by selecting only the right fields where the cells are in monolayer. Many different models of microscopes are in use today. The field diameter and the area of field viewed differ between the different models of microscopes. Since the number of platelets counted depends on the extent of area counted, the platelet count estimated by these two methods will differ between microscopes. Although the $p$ value showed a statistically significant difference, both these methods had a good correlation coefficient $(0.944)$ with automated platelet counts. This suggests that some modifications to this method might yield better results.

Method D based on the patients hemoglobin value and the average number of platelets per oil immersion field also differed significantly from the automated platelet count ( $\mathrm{p}$ value of 0.018 ). The scatterplots showed a wider range of dispersion when compared to the other three methods (Figs 1-4). This is in contrast to the results of Torres et al ${ }^{[17]}$. A study by Malok et al ${ }^{[18]}$ also showed that this method did not correlate well with the automated platelet count.

\section{Conclusion}

Thus our study results show that Method A based on measuring platelets and RBCs in ten oil immersion fields is the method of choice for estimating platelet counts in a peripheral smear if RBC count is available for the patient. This method can be used to verify the platelet counts obtained from automated hematology analyzers. However these findings need to be validated in large scale studies and by correlating with the International reference method recommended by ICSH and ILSH.

\section{Acknowledgements}

We thank all the hematology technicians of our laboratory for helping us in the study. We also thank Dr. Balaji, Assistant Professor, Department of Community Medicine for helping us in the statistical analysis.

\section{Reference}

1. De la Salle BJ, McTaggart PN, Briggs C, Harrison P, Doré CJ, Longair I, et al. The accuracy of platelet counting in thrombocytopenic blood samples distributed by the UK National External Quality Assessment Scheme for General Haematology. Am J Clin Pathol. 2012 Jan;137(1):65-74.

2. Marionneaux S, Francisco N, Chan V, Hanenberg J, Rafael J, Chua $\mathrm{C}$, et al. Comparison of automated platelet counts and potential effect on transfusion decisions in cancer patients. Am J Clin Pathol. 2013;140(5):747-54.

3. International Council for Standardization in Haematology Expert Panel on Cytometry, International Society of Laboratory Hematology Task Force on Platelet Counting. Platelet counting by the $\mathrm{RBC} /$ platelet ratio method. A reference method. Am J Clin Pathol. 2001;115(3):460-4.

4. Nosanchuk JS, Chang J, Bennett JM. The analytic basis for the use of platelet estimates from peripheral blood smears. Laboratory and clinical applications. Am J Clin Pathol. 1978;69(4):383-7.

5. Webb DI, Parker L, Webb K. Platelet count assessment from peripheral blood smear (PBS). Alaska Med. 2004;46(4):92-5.

6. Segal HC, Briggs C, Kunka S, Casbard A, Harrison P, Machin SJ, et al. Accuracy of platelet counting haematology analysers in severe thrombocytopenia and potential impact on platelet transfusion. Br J Haematol. 2005;128(4):520-5.

7. Kunz D. Possibilities and limitations of automated platelet counting procedures in the thrombocytopenic range. Semin Thromb Hemost. 2001;27(3):229-35

8. Brahimi M, Osmani S, Arabi A, Enta-Soltane B, Taghezout Z, Elkahili BS, et al. The estimation of platelet count from a blood smear on the basis of the red cell: platelet ratio. Turk J Haematol. 2009;26(1):21-4.

9. Lin J, Luo Y, Yao S, Yan M, Li J, Ouyang W, et al. Discovery and Correction of Spurious Low Platelet Counts due to EDTA-Dependent Pseudothrombocytopenia. J Clin Lab Anal. 2015;29(5):419-26.

10. Latif S, Veillon DM, Brown D, Kaltenbach J, Curry S, Linscott AJ, et al. Spurious automated platelet count. Enumeration of yeast forms as platelets by the cell-DYN 4000. Am J Clin Pathol. 2003;120(6):882-5.

11. Boulassel M-R, Al-Farsi R, Al-Hashmi S, Al-Riyami $\mathrm{H}$, Khan H, Al-Kindi S. Accuracy of Platelet Counting 
by Optical and Impedance Methods in Patients with Thrombocytopaenia and Microcytosis. Sultan Qaboos Univ Med J. 2015;15(4):e463-8.

12. Oliveira RAG, Takadachi MM, Nonoyama K, Barretto OC de O. Is automated platelet counting still a problem in thrombocytopenic blood? Sao Paulo Med J. 2003;121(1):19-23.

13. Harrison P, Segal H, Briggs C, Murphy M, Machin S. Impact of immunological platelet counting (by the platelet/RBC ratio) on haematological practice. Cytometry B Clin Cytom. 2005;67(1):1-5.

14. Abid BF. Estimation of Platelet Count on the Basis of Red cell: Platelet Ratio. Iraqi Journal of Medical Sciences. 2009;7(3):40-5
15. Gao Y, Mansoor A, Wood B, Nelson H, Higa D, Naugler C. Platelet count estimation using the CellaVision DM96 system. J Pathol Inform. 2013;4:16.

16. Bajpai R, Rajak C, Poonia M. Platelet estimation by peripheral smear: Reliable, rapid, cost effective method to assess degree of thrombocytopenia. International Journal of Medical Science Research and Practice 2015;2(2):90-93

17. Torres SL, Velez EL. Platelet verification under microscope calculated by the patient's hemoglobin factor. Laboratory Medicine 2004;7:430-3

18. Malok M, Titchener EH, Bridgers C, Lee BY, Bamberg R. Comparison of two platelet count estimation methodologies for peripheral blood smears. Clin Lab Sci. 2007;20(3):154-60.

*Corresponding author:

Dr. Muthu Sudalaimuthu, 804, New B Block, SRM Medical Staff Quarters, Potheri, kancheepuram district, Tamilnadu, India - 603203.

Phone: +91 9488070695

Email: drmuthus@gmail.com

Date of Submission : 13.11.2016

Date of Acceptance : 03.12.2016

Financial or other Competing Interests: None.

Date of Publication : 19.02.2017 